Copyright 2005 Society of Photo-Optical Instrumentation Engineers.

This paper was (will be) published in [add journal or proceedings bibliographic information] and is made available as an electronic reprint (preprint) with permission of SPIE. One print or electronic copy may be made for personal use only. Systematic or multiple reproduction, distribution to multiple locations via electronic or other means, duplication of any material in this paper for a fee or for commercial purposes, or modification of the content of the paper are prohibited. 


\title{
Imaging Study of Positive and Negative Tone Weak Phase-shifted 65 nm Node Contacts
}

\author{
James V. Beach ${ }^{1}$, John S. Petersen ${ }^{2}$, Robert T. Greenway ${ }^{2}$, Mark John Maslow ${ }^{2}$, Susan S. \\ MacDonald $^{3}$, Lee H. Margolis ${ }^{3}$, Gregory P. Hughes ${ }^{3}$ \\ ${ }^{1}$ ATDF, 2706 Montopolis Dr., Austin, TX 78741 \\ ${ }^{2}$ Petersen Advanced Lithography, 12325 Hymeadow Dr., Suite 2-201, Austin, TX 78750 \\ ${ }^{3}$ Dupont Photomask Inc., 400 Texas Ave., Round Rock, TX 78664
}

\begin{abstract}
$\mathrm{CPL}^{\mathrm{TM}}$ and aerial image mapping type contact designs for both negative and positive tones were created, built and tested for $100 \mathrm{~nm}$ and sub-100 nm contacts. Experimental results illustrated the need for electromagnetic-field corrections in the simulations. Resolution down to $80 \mathrm{~nm}$ dense contacts were seen with both negative and positive resists with acceptable process windows though some process optimization is still required as unacceptable CD variation and a reentrant profile was observed. High MEEF requires strict $\mathrm{CD}$ control on the mask. Data volume for the isolated contact designs can also challenge the mask build.
\end{abstract}

\section{INTRODUCTION}

In the comparison of SIA/ITRS roadmaps from 1995 to the present, a predicable trend can be extracted. If one tracks the dimensions of the minimum dense line, isolated line and contact sizes of the shrinking technology nodes, one will see that isolated lines grow smaller relative to the dense lines while the contact size grows larger (Table 1). Such a trend is predictable considering the asymmetry of the image log slope (NILS) over a chrome edge. The NILS of the image is larger on the darker edge than the lighter edge. Thus, overexposing an image leads to larger process windows ${ }^{1}$. Since positive resists are almost universally used in semiconductor processing, isolated lines can easily be made smaller by overexposure. Small contacts and vias, on the other hand, do not have such a simple pathway and have presented a challenge to lithographers.

\begin{tabular}{l|lllll} 
& 1995 & 1997 & 1999 & 2001 & 2003 \\
\cline { 2 - 6 } $\begin{array}{l}\text { Feature Size } \\
\text { isolated lines }\end{array}$ & 350 & 200 & 140 & 90 & 65 \\
dense lines & 350 & 250 & 180 & 130 & 100 \\
contacts & 400 & 280 & 200 & 165 & 130 \\
\hline Ratios & & & & & \\
dense:isolated & 1.00 & 0.80 & 0.78 & 0.69 & 0.65 \\
dense:contacts & 1.14 & 1.12 & 1.11 & 1.27 & 1.30
\end{tabular}

Table 1. Trends in the SIA/ITRS roadmap.

Traditionally, lithographers have turned to attenuated phase shift masks to make sub-wavelength contacts $^{2,3}$. Recently, Chromeless Phase Lithography $\left(\mathrm{CPL}^{\mathrm{TM}}\right)^{4}$ and Interference Mapping Lithography $\left(\mathrm{IML}^{\mathrm{TM}}\right)^{5}$ have offered more aggressive approaches for printing small contacts which may address some of the through-pitch issues seen with the purely attenuated phase shift approach. 
Use of negative resists could offer another approach to sub-wavelength contacts by accessing the same improvement in NILS through overexposure that isolated lines have enjoyed with positive resists. Negative resists, however, have been plagued by poor resolution, swelling and resist micro-bridging. New negative-tone $193 \mathrm{~nm}$ resists have tried to sidestep these pitfalls by abandoning the old chemical crosslinking strategy for something more similar to that of positive chemically amplified resists $6,7,8$. Though much more work has yet to be done, these new negative resists have demonstrated resolution down to $90 \mathrm{~nm}^{9}$.

As the target size of contacts shrink under $100 \mathrm{~nm}$, it seems logical to explore the application of negative tone CPL for low $\mathrm{k}_{1}$ contacts. This paper will examine CPL and aerial image mapping type contact designs for both negative and positive tones for $100 \mathrm{~nm}$ and sub-100 nm contacts. Simulations will be compared to empirical results. Challenges with mask fabrication will also be addressed.

\section{APPROACH}

The use of negative type contacts has most recently been proposed by Levenson ${ }^{10,11}$ using the Vortex contact and has been further promoted in the work of $\mathrm{Liu}^{12}$. Research at PAL, published elsewhere ${ }^{13}$, shows that the use of azimuthally polarized sources, negative resist and CPL contacts provide extensibility to $50 \mathrm{~nm}$ contacts on pitches from $90 \mathrm{~nm}$ and larger. This image design system has NILS greater than 2 and as high as 3 to 4 . More importantly, the designs keep the intensity minimum acceptably low with loss of focus and, even though it does not preclude their use, does it without the use of multiple phase regions. While, proper polarization is key for across-pitch solutions there is much to be learned with today's imaging systems.

Creating the desired contact hole image requires knowledge of the exposure tool, the feature pitch and target size, the 3D-shape of the mask after fabrication of all the features, the imaging contrast and bias of the resist for each feature and the optical properties of the wafer stack. During the design phase of this mask, we had little a priori knowledge of the resists and the mask fabrication process so ideal masks and resists were used for all simulation. For dense patterns, global biasing was used to shape the desired image. For the positive structures, two corrections were made. The first correction used Kirchoff approximation of the mask combined with vector model image formation into a lump-parameter-model (LPM) resist (thickness $=200 \mathrm{~nm}$, contrast $=17$, absorbance $=0.9 \mu \mathrm{m}^{-1}$, diffusion $=4 \mathrm{~nm}$ and $\mathrm{E} 0=15 \mathrm{~mJ} \cdot \mathrm{cm}^{-2}$ ). This was followed by making a final 3D electromagnetic-field (EMF) correction. This last correction was done using an electromagnetic-field solver (KLA-Tencor's EMF1) in off-axis mode to correct the already Optical Proximity Corrected (OPC) mask combined with the same vector and LPM models as used with the initial Kirchoff correction. PROLITH ${ }^{\mathrm{TM}}$ version 8.1.2 (KLA-Tencor) and ProLE ${ }^{\mathrm{TM}}$ version 3.1 (Petersen Advanced Lithography Inc.) were used to do the simulations.

For this study, the negative-tone structures did not receive EMF, only OPC. Instead, bracketing of the feature sizes was used in hopes of finding acceptable solutions that could be used to fingerprint the resist materials for future work.

For isolated features, an aerial image mapping technique was used to locate side lobes and to then place assist features at those locations. Phase-shifted-clear, clear and opaque assists provided Fourier components to either add energy for positive contacts or to take it away for the negatives and to increase image contrast. Knowledge gained from EMF correction at smaller pitches was applied to larger pitch structures so that a finer simulator xyz-mesh could be used to determine the necessary corrections. Large mesh checks were made but because the object size in lithographic cases is small and does more to determine an accurate EMF-solver mesh than the wavelength (meaning that a needed $2 \mathrm{~nm}$ correction is not observed if the mesh is $20 \mathrm{~nm}$ ) the results were not used to make fine corrections.

Whenever possible, care was taken to match the amplitudes of the zero-order field with that of the diffraction order with the nearest symmetric-interference angle about the optical axis and to dampen others that were not symmetric ${ }^{14}$. Whether this occurs in reality would ultimately be at the mercy of the mask fabrication and exposure tool conditions. Initial experimental results show that the EMF correction across pitch range was eight times narrower than the OPC-only range suggesting that, with proper fingerprinting of the mask and exposure tool, much tighter feature size control should be possible. Examples of the feature primitives are shown in Figure 1. 

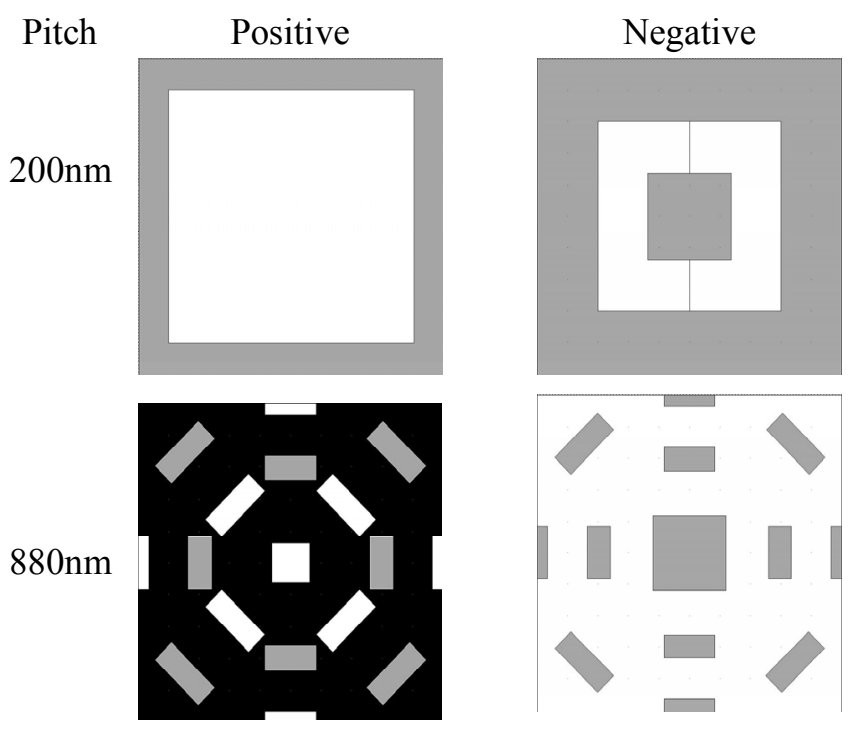

Figure 1. Examples of positive and negative resist contacts. The dark areas are chrome, the gray areas are cut quartz phase-shifters and the clear areas are uncut quartz.

\section{RETICLE MANUFACTURING}

The CPL contact design presents several reticle manufacturing challenges not present in traditional reticle manufacturing of contacts using embedded attenuated phase shift masks or more traditional binary OPC contacts. Some of these challenges include file size and figure count, lithography write time, and second level patterning for quartz etch.

The contact test reticle built for this study contained both chrome-less phase shift and hybrid designs. The dense contacts use a chrome-less phase shift design while isolated contacts use an alternating aperture phase shift design with chrome. Both designs can be manufactured using a traditional alternating aperture phase shift reticle process, with an additional final chrome removal step to form the chrome-less phased contacts. In the dense $90 \mathrm{~nm}$ contact with $180 \mathrm{~nm}$ pitch (Figure $2 \mathrm{a}$ ), the grey region is the $180^{\circ}$ phased region, and the white is the $0^{\circ}$ region. All the $180^{\circ}$ regions are opened with the first lithography write and chrome etch.

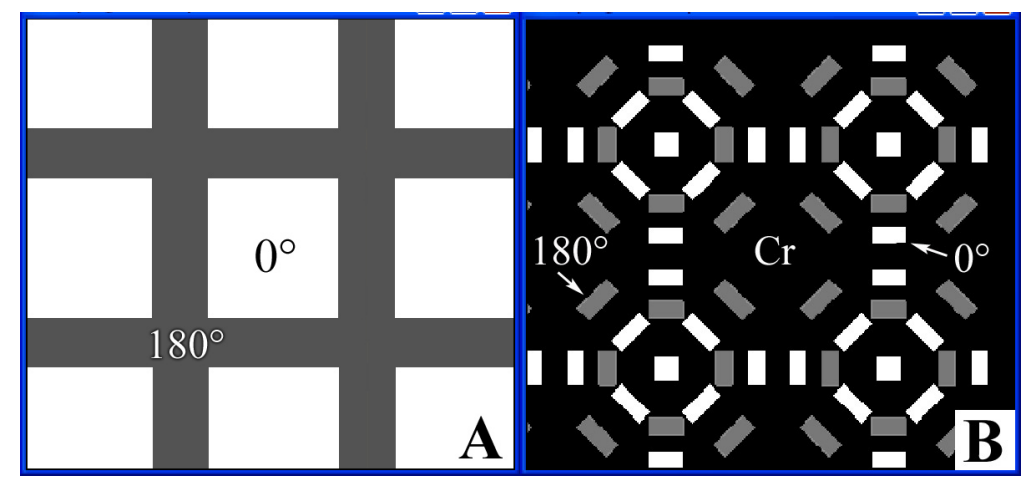

Figure 2. Sample designs of $90 \mathrm{~nm}$ dense (A) and isolated (B) contacts.

The isolated contact example is a $90 \mathrm{~nm}$ contact with a $1000 \mathrm{~nm}$ pitch (Figure $2 \mathrm{~b}$ ). The white structures are $0^{\circ}$ openings, the grey structures are $180^{\circ}$ openings, and the black background is chrome. 
Both the $0^{\circ}$ and $180^{\circ}$ structures are opened with the first lithography write and chrome etch. The minimum chrome space in this example is $100 \mathrm{~nm}$ at the reticle scale.

The first lithography is performed using a $50 \mathrm{keV}$ Vector Shaped E-beam (VSB) system. All areas that are either binary or phased are imaged. Chrome etch, and metrology is performed. The reticle is then imaged using an Alta 4300 DUV laser lithography system (A4300) to open all structures that will be quartz etched to achieve $180^{\circ}$ phase shift. After the quartz etch step is completed, a third lithography is performed to open up the chrome-less regions for chrome removal $\left(0^{\circ}\right.$ areas of Figure $\left.2 \mathrm{a}\right)$. This can be done on any laser tool with overlay capability.

A write time driver for any VSB tool and A4300 tool is file size and area coverage. The A4300 is less sensitive to this then the VSB. The main write time driver for the VSB tools is shot count. The nature of this CPL contact design will significantly increase both shot count and file size. An area of $12 \mathrm{~mm} x$ $17.5 \mathrm{~mm}$ containing arrays of isolated contacts was fractured. The total number of isolated contacts was 13.1 million, with a data density of $2 \%$, and file size of $2.6 \mathrm{MB}$. The same array with the $0^{\circ}$ and $180^{\circ}$ assist slots (Figure 3) added 8 rectangles and a minimum of 24 trapezoids per contact. This resulted in 437.1 million figures with a data density of $16.6 \%$ and file size of $44.8 \mathrm{MB}$. During the VSB write, an orthogonal $\leq 2 \mu \mathrm{m}$ contact translates to a single shot. Angled lines are created with small overlapping shots, so a single trapezoid can result in multiple shots. The exact number depends on the size and shape of the trapezoid.

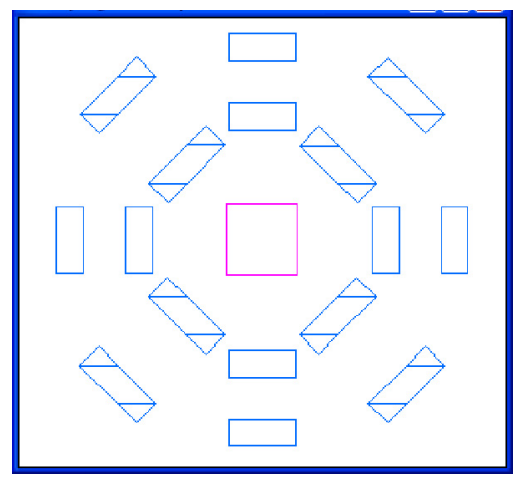

Figure 3. $90 \mathrm{~nm}$ isolated contact design broken into polygons.

The next data and lithography consideration is the second level write to open the regions for quartz etch. This reticle was manufactured using the A4300 as the second level lithography tool. It has been reported that some of the VSB tools also have overlay capability. However, when comparing the two lithography platforms, both have different concerns. Fortunately this design was completely self-aligned and minimum clear openings of the second level data made the use of the A4300 very acceptable. All quartz etch openings had chrome and some resist as the etch barrier. The only overlay accuracy constraint was opening up the $180^{\circ}$ assist slots with less then $100 \mathrm{~nm}$ of chrome between the $180^{\circ}$ structure and the $0^{\circ}$. The final reticle images of the $90 \mathrm{~nm}$ contact $1000 \mathrm{~nm}$ pitch are shown in Figure 4.

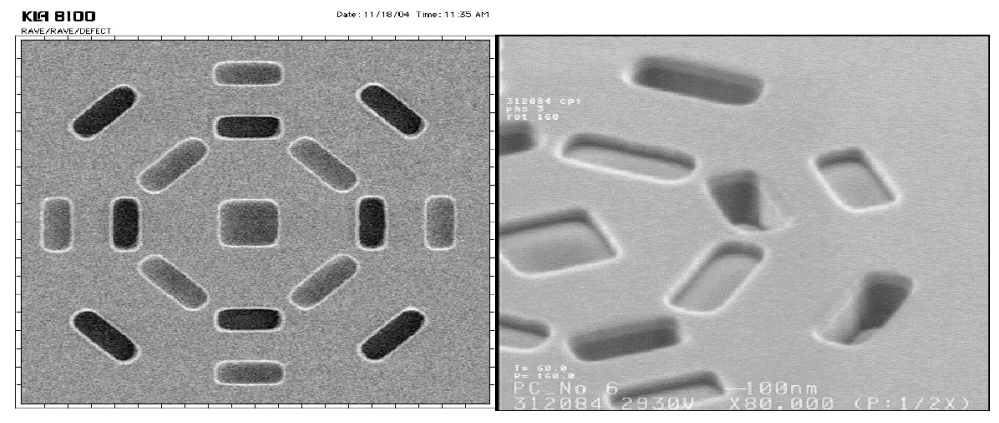

Figure 4. Top-down SEM images of the final etched mask ( $90 \mathrm{~nm}$ isolated contact). 


\section{RESULTS AND DISCUSSION}

All lithography was performed on bare silicon wafers using Brewer Science ARC-29 as a bottom anti-reflective coating (BARC). All images were shot using a 0.75 NA $193 \mathrm{~nm}$ scanner using either 0.89/0.65 Quasar illumination or a $0.92 / 0.72$ cross-quadrupole ( $45^{\circ}$ rotated Quasar). Resist thickness for both the positive and negative resist was $2400 \AA$. Both positive and negative approaches were successful at resolving all designs targeted in this paper except for the isolated contacts for the negative resist. This failure will be discussed later.

A couple of general items were noticeable immediately from the top-down SEM images of the contacts in resist (Figure 5). The smaller contacts $(<100 \mathrm{~nm})$ were not perfectly round but had a certain amount of random roughness to the shape. Qualitatively, the negative resist showed a better roundness than the positive resists tried. The contact hole shape may be tied to line-edge roughness and will be addressed in a separate paper.
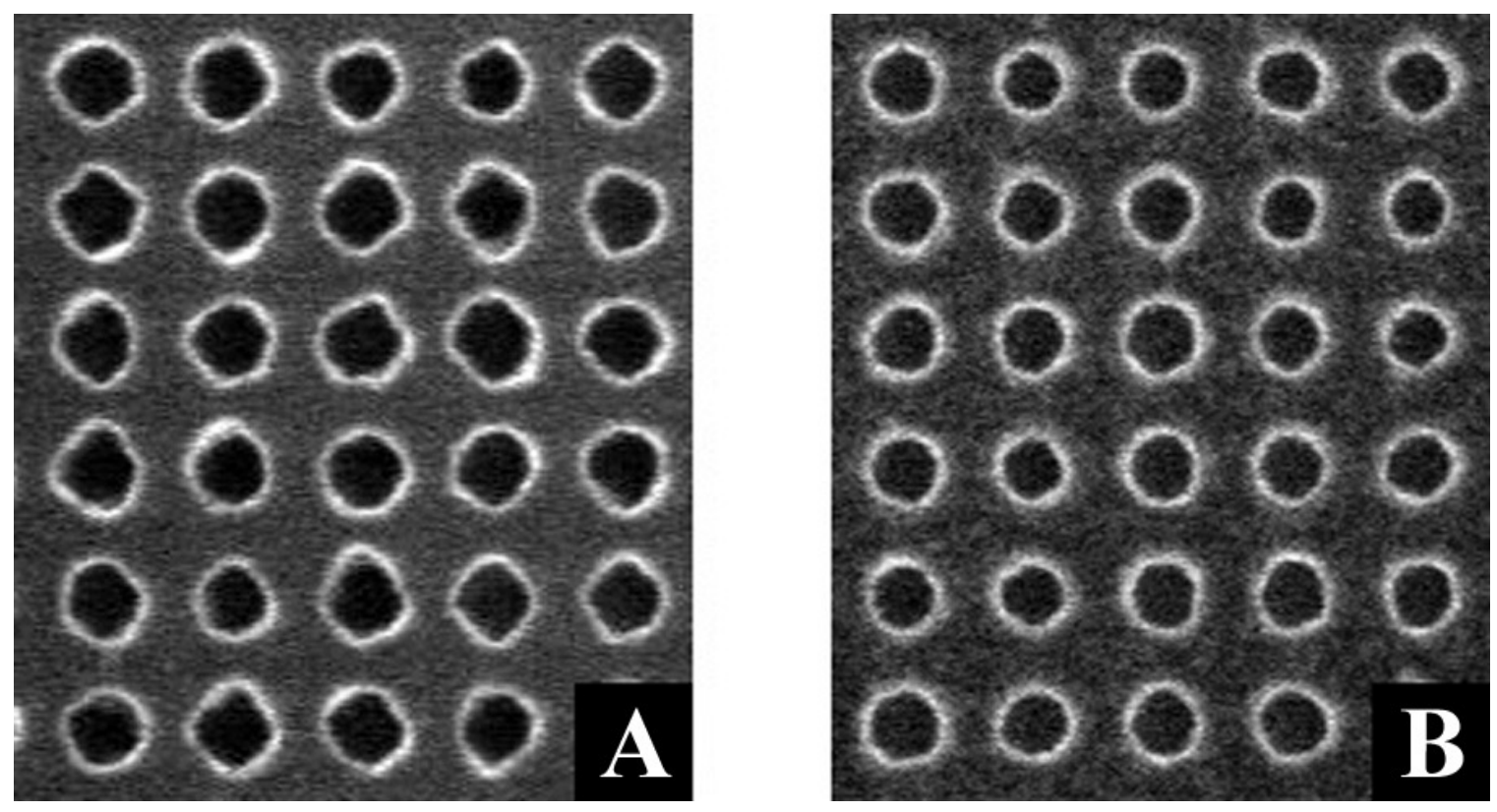

Figure 5. Top down images of $80 \mathrm{~nm}$ contacts on a $160 \mathrm{~nm}$ pitch for positive (A) and negative (B) resist.

A second issue that is apparent from Figure 5 is that there is a small variation in contact hole size. This variation could be a result of either the resist performance or may be linked to the mask itself as the MEEF is known to be high. Table 2 shows the range and standard deviation for the critical dimensions (CD) measured for different contact types at best does/focus. Data shown represents the contacts shot with cross-quadrupole illumination. A sample size of 20 measurements per feature was used. One can see a trend of the absolute range and standard deviation increasing as the feature size decreases. In many cases, 6-sigma variation would be larger than a $+/-10 \%$ spec window making the process unsuitable for production without further process optimization. 


\begin{tabular}{|c|c|c|c|c|}
\hline \multirow{4}{*}{ negative } & & ave & range & std. \\
\hline & $80 / 160$ & $77.0 \mathrm{~nm}$ & $19 \mathrm{~nm}$ & 5.6 \\
\hline & $90 / 180$ & $88.4 \mathrm{~nm}$ & $20 \mathrm{~nm}$ & 5.4 \\
\hline & $100 / 200$ & $101.4 \mathrm{~nm}$ & $17 \mathrm{~nm}$ & 3.6 \\
\hline \multirow[t]{6}{*}{ Positive } & $80 / 160$ & $80.4 \mathrm{~nm}$ & $18 \mathrm{~nm}$ & 4.4 \\
\hline & $90 / 180$ & $88.7 \mathrm{~nm}$ & $12 \mathrm{~nm}$ & 3.4 \\
\hline & $100 / 200$ & $100.8 \mathrm{~nm}$ & $8 \mathrm{~nm}$ & 3.2 \\
\hline & $80 / 880$ & $81.2 \mathrm{~nm}$ & $13 \mathrm{~nm}$ & 3.3 \\
\hline & $90 / 1000$ & $92.5 \mathrm{~nm}$ & $8 \mathrm{~nm}$ & 1.8 \\
\hline & $100 / 1100$ & $102.4 \mathrm{~nm}$ & $6 \mathrm{~nm}$ & 1.6 \\
\hline
\end{tabular}

Table 2. Statistical data for contact size variation.

Experimental results clearly demonstrated the necessity of the EMF corrections for the contact designs described in the approach section of this paper. Figure 6 shows the experimentally determined dose-to-size of the $80 \mathrm{~nm}, 90 \mathrm{~nm}$ and $100 \mathrm{~nm}$ isolated and dense contacts (positive resist) for both the Kirchoff and EMF solutions. The Kirchoff designs yield extreme differences in dose-to-size for the dense and isolated contacts offering no hope for overlapping process windows. With EMF corrections, however, the dose-to-size for the different pitches are clustered much more closely and could likely be moved even closer together with small adjustments to the mask design by using a more complete mask transform. Unless stated otherwise, all data presented in this paper for the positive contacts are from designs using the EMF solution. Unfortunately, contact designs with EMF corrections are present only for the positive-resist solutions on this reticle.

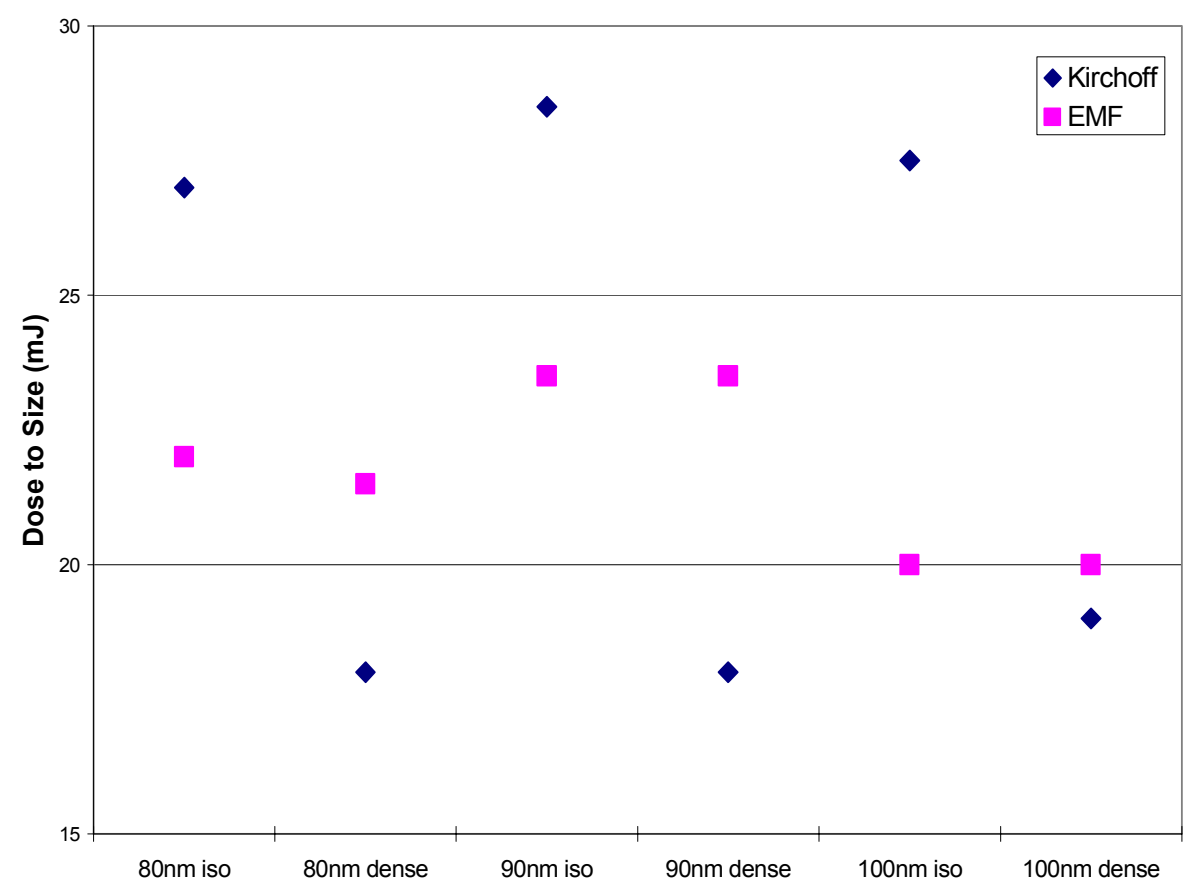

Figure 6. Empirical dose-to-size of Kirchoff and EMF contact designs. 
As predicted by simulation, pitches below $200 \mathrm{~nm}$ did not resolve well using Quasar illumination $(0.89 / 0.65)$. The $100 \mathrm{~nm}$ contacts on a $200 \mathrm{~nm}$ pitch did, however, show a relatively wide process window for the positive resist contacts and a marginal process window for the negative contacts (Figure 7). All designs on the reticle were optimized for use with a cross-quadrupole and, therefore, a direct comparison of the two systems under Quasar illumination may not be fair. The experimental results do follow closely to what is expected by simulation with the exception of the smaller observed exposure latitude for the negative resist. This observation is most likely a result of inaccurate negative resist-model parameters and, possibly, the lack of a proper mask transform for the center assist feature in the negative contact.
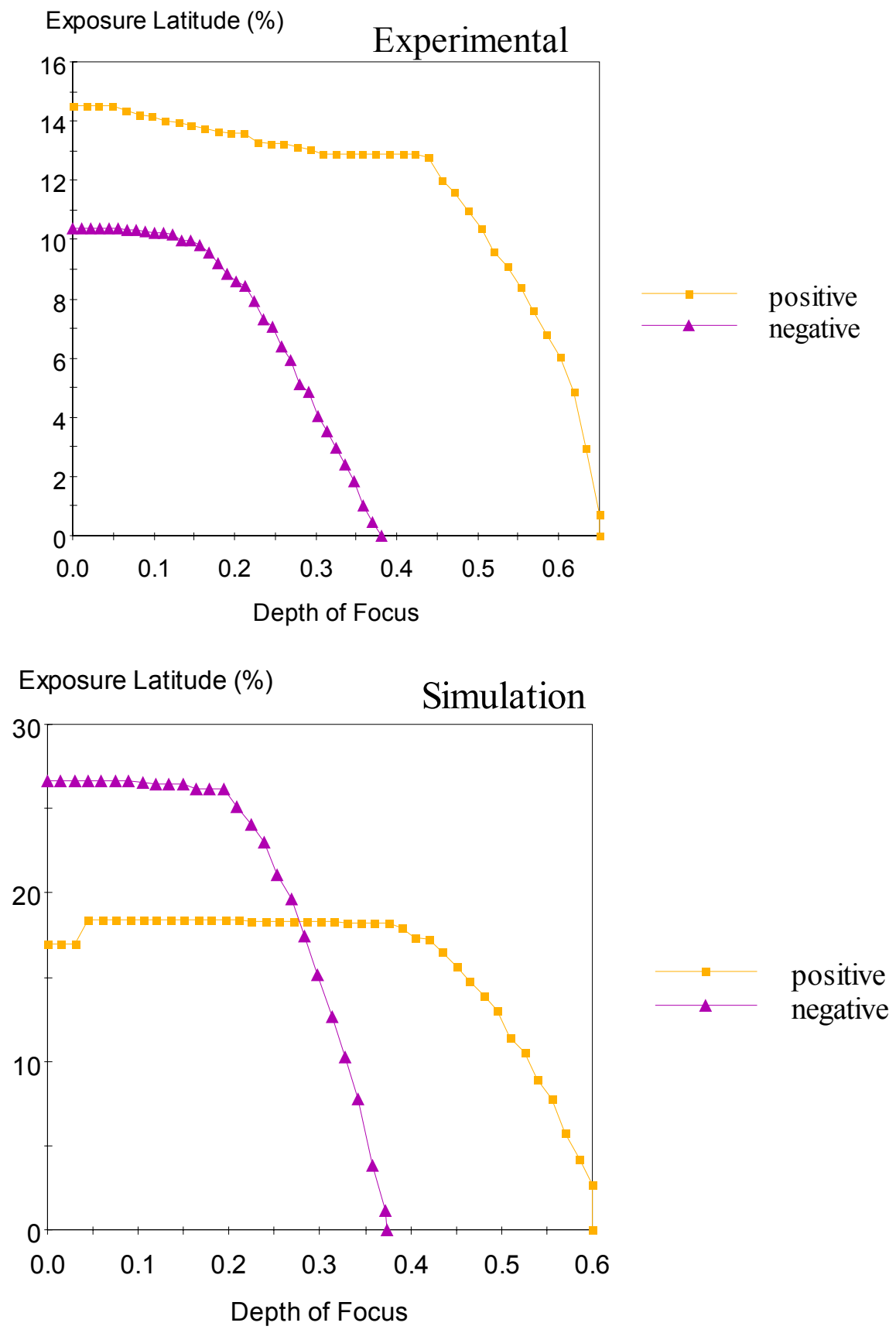

Figure 7. Empirical and simulated process windows for $100 \mathrm{~nm}$ dense contacts using 0.89/0.65 Quasar illumination. 
Use of a cross-quadrupole (0.92/0.72) allowed resolution of contacts at a $180 \mathrm{~nm}$ and $160 \mathrm{~nm}$ pitch but resulted in a drastic reduction of the process windows at a $200 \mathrm{~nm}$ pitch as this pitch becomes forbidden under these illumination conditions. The process windows improve as the pitch moves to 160 $\mathrm{nm}$ (Figure 8). At each pitch, the positive resists out performed the negative resists by a small margin. This, again, could be a reflection of the EMF optimization present in the positive resist designs or could be a reflection of the maturity of positive resists over the negative resist.

\section{Exposure Latitude (\%)}

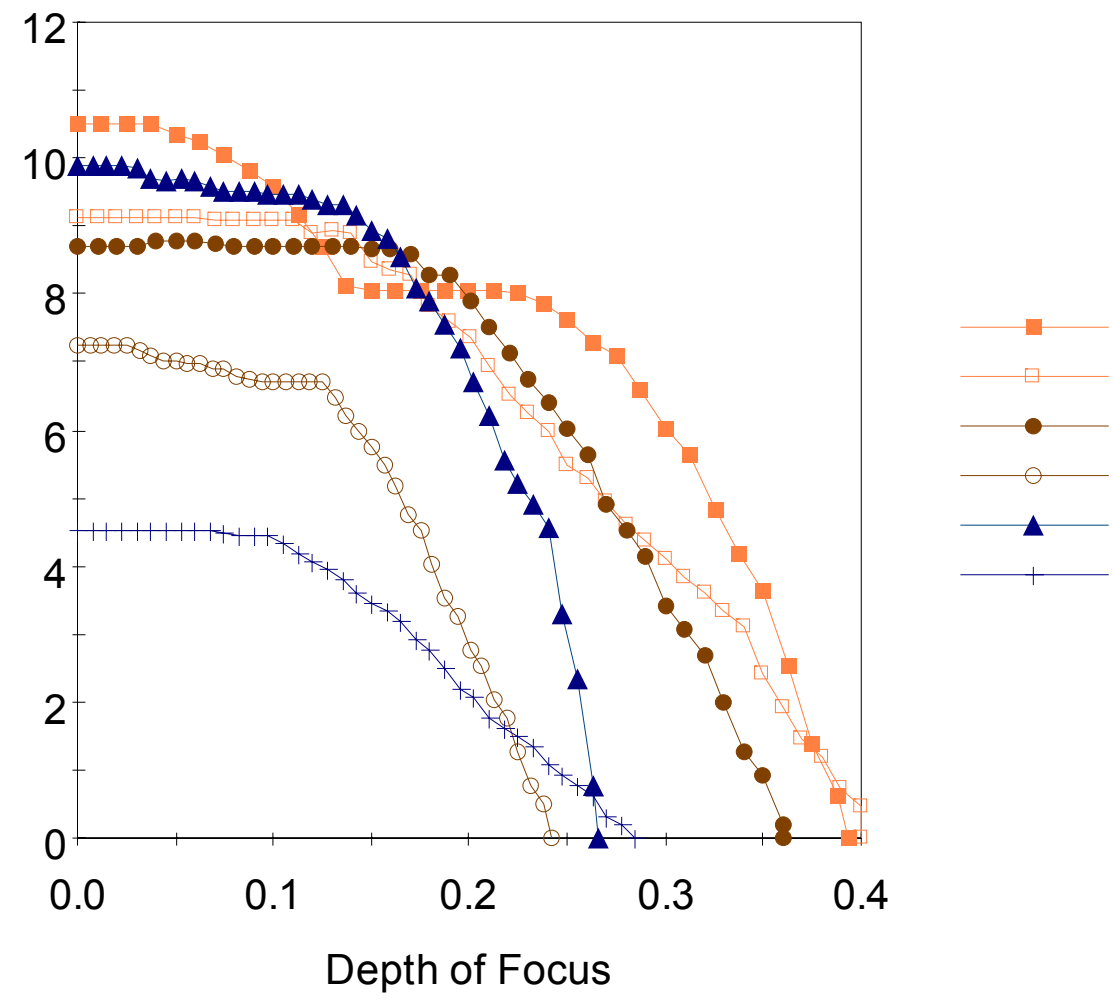

positive $80-160$

negative $80-160$

positive $90-180$

negative $90-180$

positive $100-200$

negative $100-200$

Figure 8. Empirical process windows for the CPL dense contacts.

For the positive resist, the isolated $80 \mathrm{~nm}$ contacts show a very small process window. The $90 \mathrm{~nm}$ and 100 $\mathrm{nm}$ isolated contacts showed much larger process windows (Figure 9). Unfortunately, the negative resist showed no process window for any of the isolated contacts. Revisiting the simulations revealed a surprising result when EMF corrections were applied to the Kirchoff solutions. Figure 10 shows how the aerial image drastically changes with the EMF solution and the contact looses depth of focus. Therefore, the bracketing strategy attempted on this mask failed to yield a functional contact design. This result, again, illustrates the necessity of electromagnetic-field corrections in the simulations of small contacts. The results are most evident for the isolated negatives but simulation shows that the 3D Mask Transform ${ }^{13}$ is significantly different for negative contacts than for positive. The result is reflected in Figure 10 showing a phase inversion that causes the image to flip from post to open with change in focus that is not observed with Kirchoff. Figure 11 shows the observed overlapping process windows for all the positive contacts with EMF correction. Considering that the designs assumed ideal mask, resist and exposure tool properties, the overlap is very good. Only the $80 \mathrm{~nm}$ dense and isolated contacts fell significantly out of the overlapping region. 


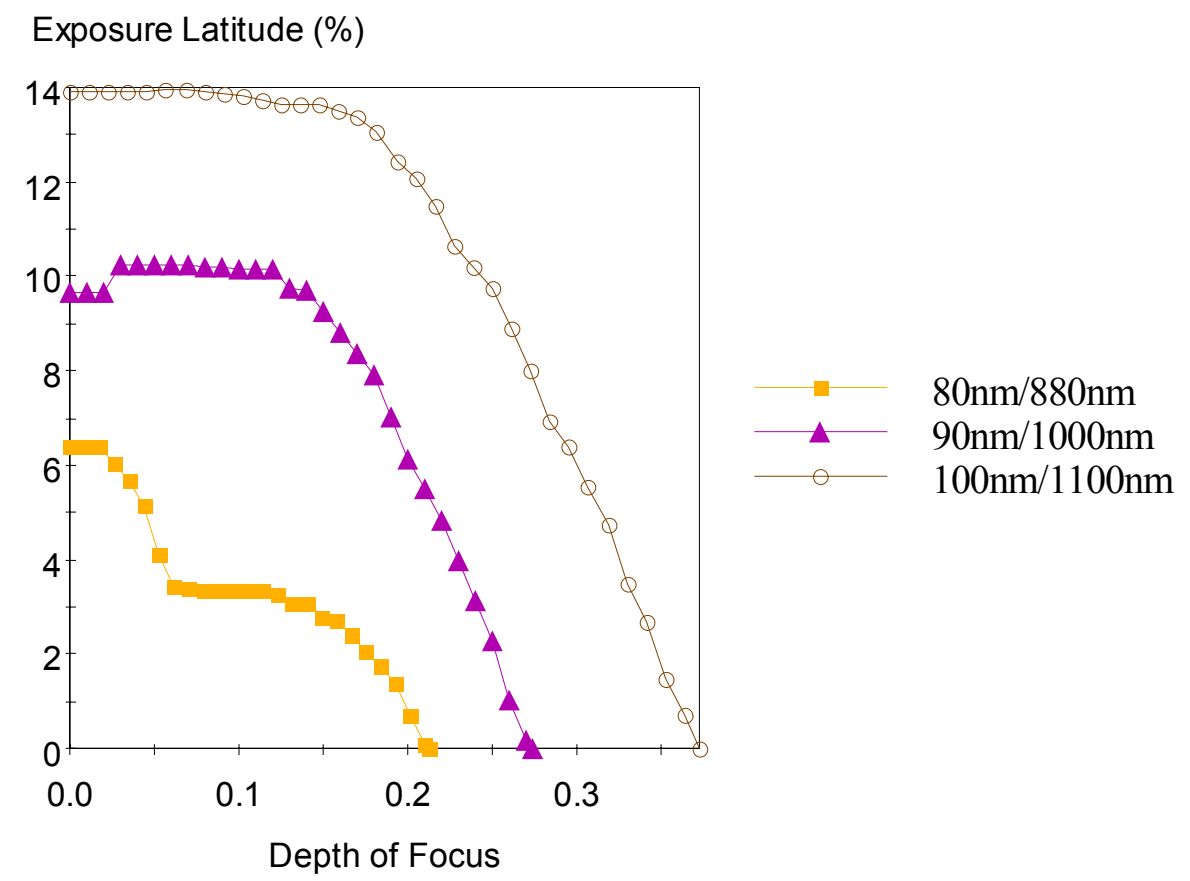

Figure 9. Process windows for the isolated contacts (positive resist only).
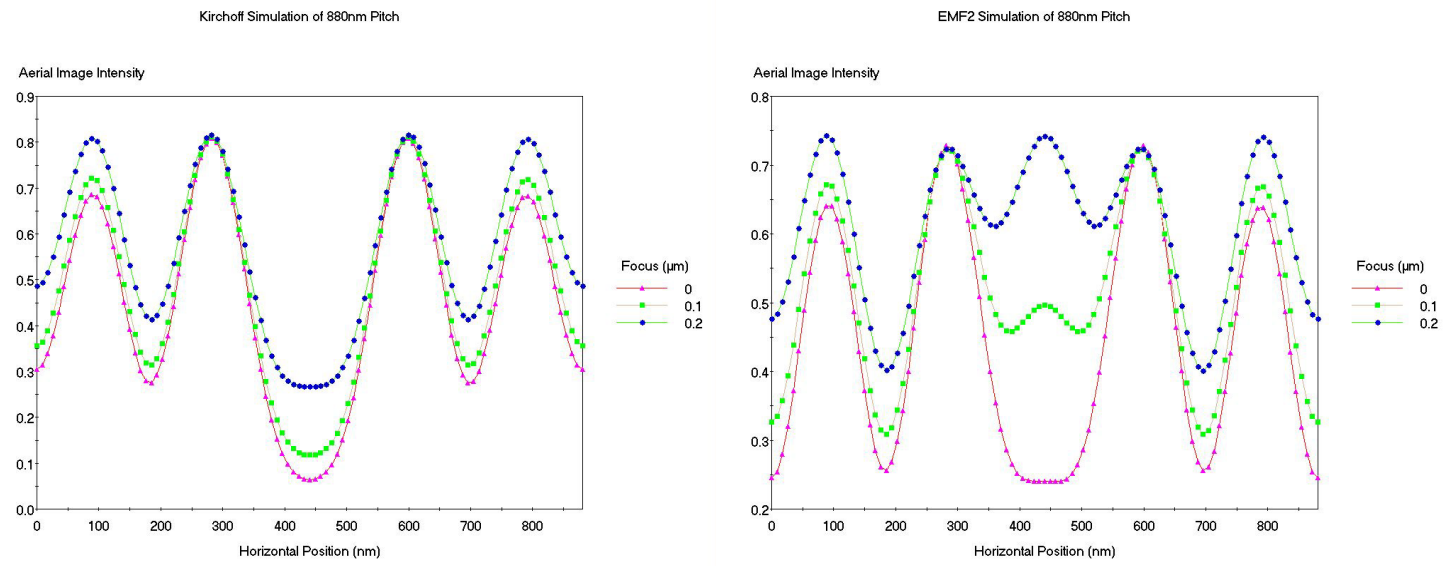

Figure 10. Aerial image comparison of a negative isolated contact using Kirchoff vs. EMF simulations. 


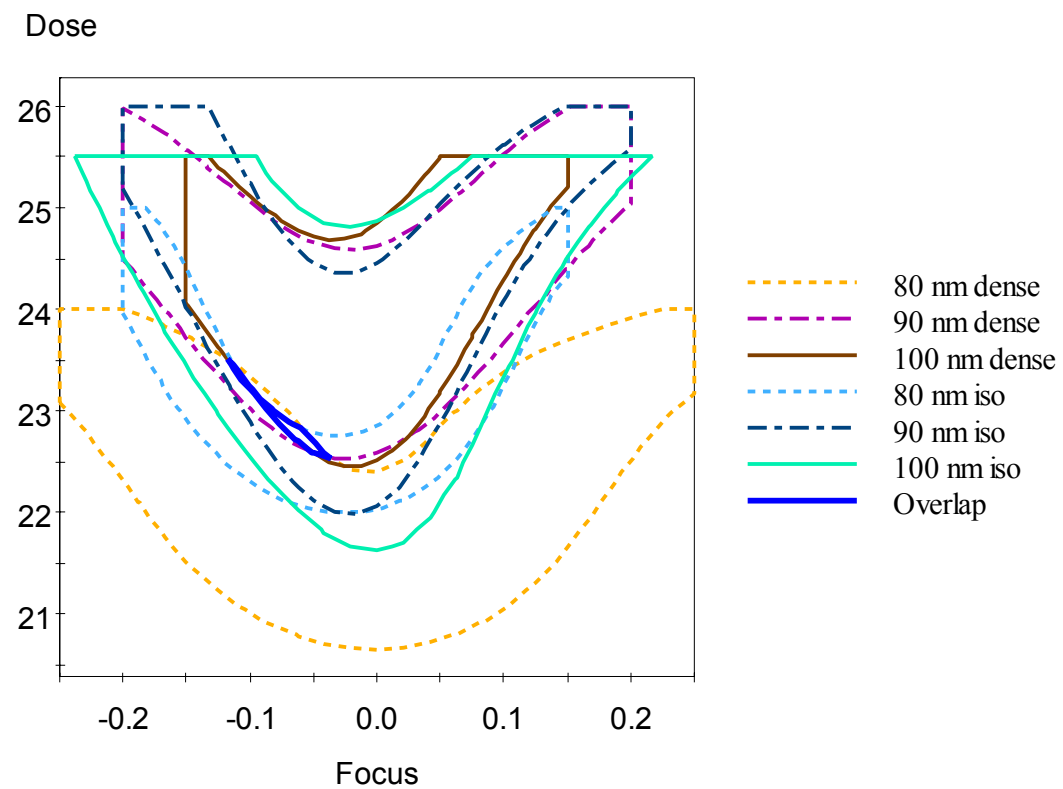

Figure 11. Experimentally determined overlapping process windows of the EMF-corrected positive contacts (isolated and 1:1 dense).

Cross sections show a reentrant profile for all contacts shot with the cross-quadrupole (Figure 12). It is possible the higher angle of the cross-quadrupole requires re-optimization of the BARC and resist thicknesses to improve the standing waves. This profile is not seen when the mask was shot with Quasar illumination. It is interesting to note that the negative resist, again, showed a smoother profile than the positive resist.

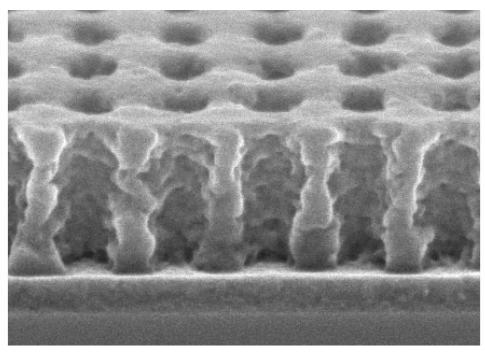

$90 \mathrm{~nm} \mathrm{1:1} \mathrm{(positive} \mathrm{resist)}$

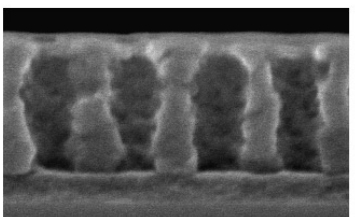

$80 \mathrm{~nm} \mathrm{1:1} \mathrm{(positive)}$

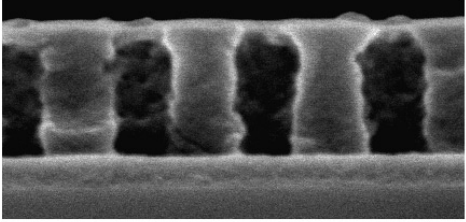

$100 \mathrm{~nm} \mathrm{1:1} \mathrm{cross-quad}$

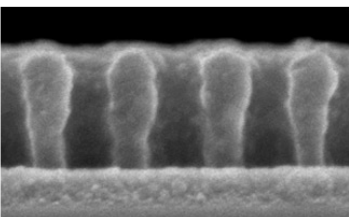

$80 \mathrm{~nm} \mathrm{1:1} \mathrm{(negative)}$

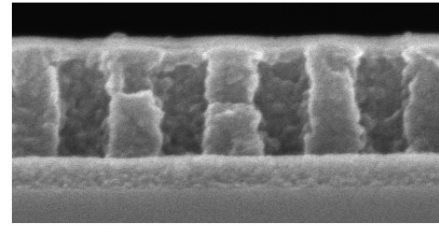

$100 \mathrm{~nm} \mathrm{1:1} \mathrm{Quasar}$

Figure 12. Cross-sectional view of isolated and dense contacts. 
Although CPL contacts show impressive results, MEEF is known to be an issue requiring very tight $\mathrm{CD}$ tolerances for the mask makers. MEEF was measured by comparing the top-down SEM sizing of the patterned contacts of the bracketed designs. Mask CD measurements were taken in each bracket to insure an accurate number was used for the mask error. As expected, MEEF is high $(>3.0)$ for both the dense and isolated designs (Table 3). There does not appear to be any significant difference between the performance of the negative and positive contacts.

\begin{tabular}{ll|c}
\multicolumn{1}{c}{} & \multicolumn{2}{c}{ MEEF } \\
\cline { 3 - 3 } Negative & $80 / 160$ & 5.7 \\
& $90 / 180$ & 3.0 \\
& $100 / 200$ & 8.5 \\
\hline Positive & $80 / 160$ & 3.0 \\
& $90 / 180$ & 7.6 \\
& $100 / 200$ & 5.0 \\
& $80 / 880$ & 5.9 \\
& $90 / 1000$ & 4.7 \\
& $100 / 1100$ & 3.8
\end{tabular}

Table 3. MEEF measurements of the different contact designs.

\section{CONCLUSION}

High resolution isolated and dense contacts were imaged through the use of CPL and aerial image mapping designs for both negative and positive tones. The manufacturing of the mask was achieved using conventional lithography tools and standard etched quartz phase shift processes. There is concern over the data volume and long write times on the VSB lithography tools. EMF corrections were found to be necessary for accurate simulation of the contact performance. Lack of EMF corrections for the negative tone isolated contacts lead to designs with zero empirical process windows. With the dense contacts, the positive system performed slightly better than the negative systems in terms of process window. This may be a reflection of both the lack of EMF optimization and the immaturity of negative resists. Contacts showed roughness in both shape and profile, though the negative resist looked qualitatively smoother. CD variation and a reentrant profile suggest that further process optimization is needed.

\section{REFERENCES}

1. Brunner, T. A.; Fonseca, C., "Optimum tone for various feature types: positive versus negative", Proc. SPIE, 4345, pp.30-49, 2001.

2. Socha, R. J.; Petersen, J. S.; Chen, F.; Laidig, T.; Wampler, K.; Caldwell, R., "Design of 200nm, 170nm, 140nm DUV Contact Sweeper High Transmission Attenuating Phase Shift Mask through Simulation Part 1", Proc. SPIE, 3546, pp. 617-641, 1998.

3. Beach, J. V.; Petersen J. S.; Eynon, B.; Tayor, D.; Gerold, D. J.; Maslow, M. J, "Imaging $100 \mathrm{~nm}$ contacts with high transmission attenuated phase shift masks", Proc. SPIE, 4889, pp.1242-1252, 2002.

4. Wiaux, V.; et. al., "ArF solutions for low-k 1 back-end imaging”, Proc. SPIE, 5040, pp. 270-281, 2003. 5. Socha, R.; et. al., "Contact Hole Reticle Optimization by Using Interference Mapping Lithography (IML ${ }^{\mathrm{TM}}$ )", Proc. SPIE, 5377, pp. 222-240, 2004.

6. Yokoyama, Y.; Hattori, T; Kimura, K; Tanaka, T. P.; Shiraishi, H., "ArF negative resist system using androsterone structure with $\delta$-hydroxy acid for 100 -nm phase shifting lithography", Proc. SPIE, 4345, pp. 58-66, 2001. 
7. Fu, S.; Hsieh, K.; Wang, L. A., "Negative-tone cycloolefin photoresist for 193-nm lithography", Proc. SPIE, 4345, pp. 751-760, 2001.

8. Sooriyakumaran, R; et. al., "193-nm negative resist based on acid-catalyzed elimination of polar molecules”, Proc. SPIE, 5376, pp. 71-78, 2004.

9. Patel, K.; et. al., "IBM-JSR 193-nm negative tone resist: polymer design, material properties, and lithographic performance”, Proc. SPIE, 5376, pp. 94-102, 2004.

10. Levenson, M. D., Dai, G., Ebihara, T. (J.), "The vortex mask: making 80nm Contacts with a twist!" Proc. SPIE, 4889, pp. 1293-1303, 2002.

11. Levenson, M. D., Ebihara, T. (J.), Morikawa, Y., Hayashi, N., "Vortex Via Validation", Proc. SPIE, 5256, pp. 93-102, 2004.

12. Liu, Y., Hu, J., Liu, D. "Single exposure general vortex phase-shift mask for contact hole", Proc. SPIE, 5567, pp 723-731, 2004.

13. Petersen, J. S., Maslow M. J., Greenway, R. T., “An Integrated imaging system for the $45 \mathrm{~nm}$ technology node contact holes using polarized off axis illumination, immersion, weak phase-shift masks and negative resists, Proc. SPIE, 5754, paper 5754-46, 2005.

14. "Method for phase shift mask design, fabrication, and use", US Patent 6,800,401, Date of Patent October 5, 2004. 